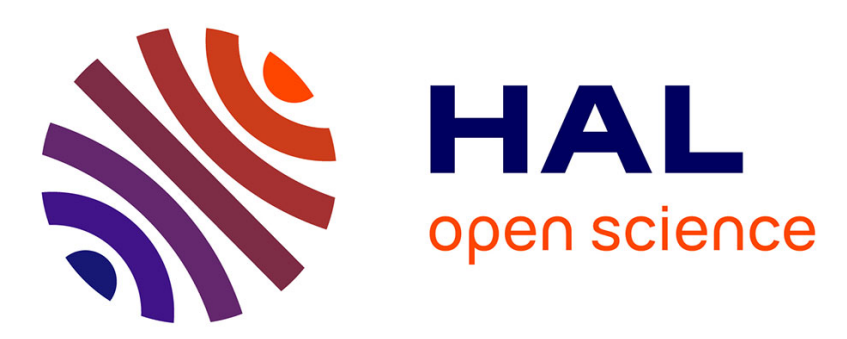

\title{
Beyond blow-up in excitatory integrate and fire neuronal networks: refractory period and spontaneous activity
}

\author{
Maria J. Caceres, Benoît Perthame
}

\section{To cite this version:}

Maria J. Caceres, Benoît Perthame. Beyond blow-up in excitatory integrate and fire neuronal networks: refractory period and spontaneous activity. Journal of Theoretical Biology, 2014, 350, pp.8189. 10.1016/j.jtbi.2014.02.005 . hal-00874746

\section{HAL Id: hal-00874746 \\ https://hal.sorbonne-universite.fr/hal-00874746}

Submitted on 18 Oct 2013

HAL is a multi-disciplinary open access archive for the deposit and dissemination of scientific research documents, whether they are published or not. The documents may come from teaching and research institutions in France or abroad, or from public or private research centers.
L'archive ouverte pluridisciplinaire HAL, est destinée au dépôt et à la diffusion de documents scientifiques de niveau recherche, publiés ou non, émanant des établissements d'enseignement et de recherche français ou étrangers, des laboratoires publics ou privés. 


\title{
Beyond blow-up in excitatory integrate and fire neuronal networks: refractory period and spontaneous activity
}

\author{
María J. Cáceres* Benoît Perthame ${ }^{\dagger}$
}

June 11, 2013

\begin{abstract}
The Network Noisy Leaky Integrate and Fire equation is among the simplest model allowing for a self-consistent description of neural networks and gives a rule to determine the probability to find a neuron at the potential $v$. However, its mathematical structure is still poorly understood and, concerning its solutions, very few results are available. In the midst of them, a recent result shows blow-up in finite time for fully excitatory networks. The intuitive explanation is that each firing neuron induces a discharge of the others; thus increases the activity and consequently the discharge rate of the full network.

In order to better understand the details of the phenomena and show that the equation is more complex and fruitful than expected, we analyze further the model. We extend the finite time bow-up result to the case when neurons, after firing, enter a refractory state for a given period of time. We also show that spontaneous activity may occur when, additionally, randomness is included on the firing potential $V_{F}$ in regimes where blow-up occurs for a fixed value of $V_{F}$.
\end{abstract}

Key words: Integrate and fire models; noise; neural networks; blow-up; spontaneous activity; Mathematics Subject Classification: 35K60, 82C31, 92B20

\section{Introduction}

The Network Noisy Leaky Integrate and Fire (NNLIF in short) model is certainly one of the simplest self-contained mean field equation for neural networks. It describes, at time $t$, the probability $p(v, t)$ to find a neuron at a voltage $v$, assuming each individual neuron follows a simple integrate and fire dynamics and the coupling changes the current. Integrate and Fire models,for a single neuron or a population of neurons, with or without noise, have been used very widely [1, 4, 20, 23, 14, 15, compared to experimental data [2, 21] and qualitative properties have been studied [3, 4, 22, 10]. Many references can be found in surveys and books, see [11, 24, 12] among others. However its mathematical structure is still poorly understood and very few results are available concerning its solutions. For instance, very recent results are large time existence for the inhibitory case [7, short time existence of smooth solutions for the excitatory case [8] and global existence for the model when firing neurons induce finite jumps 9]. Another striking mathematical property is that for fully excitatory networks, the system blows-up in finite time; this holds for any initial data for a large enough network connectivity and for any connectivity

\footnotetext{
*Departamento de Matemática Aplicada, Universidad de Granada, E-18071 Granada, Spain. Email: caceresg@ugr.es

${ }^{\dagger}$ UPMC Univ. Paris 06, CNRS UMR 7598, Laboratoire Jacques-Louis Lions, F75005 Paris and INRIA Rocquencourt EPI BANG. Email: benoit.perthame@upmc.fr
} 
if the initial data is concentrated enough near the firing potential denoted by $V_{F}$ in the sequel, see [5]. This also remains true when the discrete nature of interactions is kept 10. The intuitive explanation is that each firing neuron induces a discharge of the others; thus increases the activity and consequently the discharge rate of the full network. Finally, synchronous states, where the firing rate does not tend asymptotically to constant in time but network produces spontaneous activity, have also been observed in several neuronal networks models: systems of coupled nonlinear oscillators 11, inhibitory NNLIF with synaptic integration [4, excitatory-inhibitory coupled NNLIF [3], Fokker-Planck equations for uncoupled neurons [14, 15, kinetic models [19, 6] and elpased time models [16].

In order to better understand the details of the phenomena observed in NNLIF, we analyze a model with a refractory state. The refractory state takes into account the fact that neurons during the refractory period do not respond on stimuli. We describe the influence of that period coupling the NNLIF equation, considered in [5], with a ODE for the refractory state:

$$
\left\{\begin{array}{l}
\frac{\partial p}{\partial t}(v, t)+\frac{\partial}{\partial v}[h(v, N) p(v, t)]-a(N(t)) \frac{\partial^{2} p}{\partial v^{2}}(v, t)=\frac{R(t)}{\tau} \delta\left(v-V_{R}\right), \quad v \leq V_{F}, \\
\frac{d R(t)}{d t}=N(t)-\frac{R(t)}{\tau}, \\
N(t):=-a(N(t)) \frac{\partial p}{\partial v}\left(V_{F}, t\right) \geq 0, \\
p\left(V_{F}, t\right)=0, \quad p(-\infty, t)=0, \quad p(v, 0)=p^{0}(v) \geq 0, \quad R(0)=R^{0}>0 .
\end{array}\right.
$$

In this system, $p(v, t)$ represents the density of active neurons (those which respond on stimuli) in the network at voltage $v \in\left(-\infty, V_{F}\right), R(t)$ denotes the probability density to find a neuron in the refractory state and $N(t)$ is the flux of firing neurons. The presentation of the model ends up with the description of the parameters:

- $V_{R}$ and $V_{F}$ are the reset and the firing potentials, respectively, and $v=0$ is chosen as the relaxation potential.

- $b$ represents the connectivity of the network: $b>0$ for excitatory networks and $b<0$ for inhibitory.

- $\tau$ measures the mean duration of the refractory period.

- $h(v, N)$ is the drift coefficient and usually will be $h(v, N)=-v+b N$.

- $a(N)$ denotes the activity dependant noise; it is usual to take the form $a(N)=a_{0}+a_{1} N$ and here we just assume

$$
a(N) \geq a_{m}>0
$$

An important and deep literature has been devoted to the derivation of mean field equations as (1D) for large systems of coupled neurons, see [24, 12, 19, 8, 17, for instance.

The first property of the system (11) that one readily checks is the conservation of the total number of neurons, that is

$$
R(t)+\int_{-\infty}^{V_{F}} p(v, t) d v=R^{0}+\int_{-\infty}^{V_{F}} p^{0}(v) d v=1 .
$$


Brunel in [3] presents a different model to depict the presence of refractory state, he considers

$$
R(t)=\int_{t-\tau}^{t} N(s) d s
$$

and couple it with the equation for the active neurons in the network written as

$$
\frac{\partial p}{\partial t}(v, t)+\frac{\partial}{\partial v}[h(v, N) p(v, t)]-a(N(t)) \frac{\partial^{2} p}{\partial v^{2}}(v, t)=N(t-\tau) \delta\left(v-V_{R}\right), \quad v \leq V_{F} .
$$

The conservation property (3) still holds since we have

$$
\frac{d}{d t}\left[\int p(v, t) d v+R(t)\right]=0
$$

at least if $N$ is properly extended for times $t \in(-\tau, 0)$. This latter model considered in [3] and the system (11) are particular cases of a general version

$$
\left\{\begin{array}{l}
\frac{\partial p}{\partial t}(v, t)+\frac{\partial}{\partial v}[h(v, N(t)) p(v, t)]-a(N(t)) \frac{\partial^{2} p}{\partial v^{2}}(v, t)=M(t) \delta\left(v-V_{R}\right) \\
\frac{d R(t)}{d t}=N(t)-M(t) .
\end{array}\right.
$$

We recover the model in [3] with the choice $M(t)=N(t-\tau)$ and the model (11) with $M(t)=R(t)$. Our results and proofs below also hold within the more general setting (4) and therefore remain true for the Brunel's model in [3].

We consider throughout this paper weak solutions which definition is recalled in Section 2, Our main result is that weak solutions to (11) with $b>0$ blow-up in the same condition than when the refractory state is ignored; this result is proved in Section 3. Then we study the existence and multiplicity of steady states in Section 4, both with a general result and some numerical illustrations (see Section 7). For $b \leq 0$ there is a unique steady state and we can show that the linear equation, that is $b=0$, comes with a natural energy which explains the long time relaxation to the unique steady state (Section 5 ).

Self-sustained oscillations can also be obtained for the excitatory NNLIF when the deterministic value $V_{F}$ is changed to a random discharge potential. We study two examples of discharge laws and prove in Section [6 that solutions are a priori globally bounded. Numerical illustrations are finally given in Section 17. Section 8 summarizes the paper and gives conclusions on our results about the NNLIF with refractory state; it also presents several open questions.

\section{Notation and definitions}

In this section we present the notations that are used throughout this paper and the definition of weak solutions of (11). We recall some usual notations: $L^{p}(\Omega)$ with $1 \leq p<\infty$ is the space of functions such that $f^{p}$ is integrable in $\Omega, L^{\infty}$ represents the space of bounded functions in $\Omega$ and $C^{\infty}(\Omega)$ denotes the space of infinitely differentiable functions in $\Omega$.

Definition 2.1 Let $p \in L^{\infty}\left(\mathbb{R}^{+} ; L_{+}^{1}\left(-\infty, V_{F}\right)\right), N \in L_{\mathrm{loc},+}^{1}\left(\mathbb{R}^{+}\right)$and $R \in L_{+}^{\infty}\left(\mathbb{R}^{+}\right)$. We say that $(p, N, R)$ is a weak solution of (1I) if for any test function $\phi(v, t) \in C^{\infty}\left(\left(-\infty, V_{F}\right] \times \mathbb{R}^{+}\right)$such that $\frac{\partial^{2} \phi}{\partial v^{2}}, v \frac{\partial \phi}{\partial v} \in$ 
$L^{\infty}\left(\left(-\infty, V_{F}\right) \times \mathbb{R}^{+}\right)$we have

$$
\begin{aligned}
\int_{0}^{T} \int_{-\infty}^{V_{F}} p(v, t)\left[-\frac{\partial \phi}{\partial t}-h(v, N) \frac{\partial \phi}{\partial v}\right. & \left.-a(N) \frac{\partial^{2} \phi}{\partial v^{2}}\right] d v d t=\int_{0}^{T}\left[\frac{R(t)}{\tau} \phi\left(V_{R}, t\right)-N(t) \phi\left(V_{F}, t\right)\right] d t \\
& +\int_{-\infty}^{V_{F}} p^{0}(v) \phi(0, v) d v-\int_{-\infty}^{V_{F}} p(v, T) \phi(T, v) d v
\end{aligned}
$$

and $R$ is a solution of the $O D E$

$$
\frac{d R(t)}{d t}=N(t)-\frac{R(t)}{\tau}
$$

We point out that the growth conditions for the test functions $\phi$ is needed to give sense to the term involving $h(v, N)$ when $h(v, N)=-v+b N$. To treat a more general drift $h$, we should assume additionally that $h(v, N) \frac{\partial \phi}{\partial v} \in L^{\infty}\left(\left(-\infty, V_{F}\right) \times \mathbb{R}^{+}\right)$. It is also standard to consider test functions of the form $\psi(t) \phi(v)$; then the double integral in this weak formulation is equivalent to say that for all $\phi(v) \in C^{\infty}\left(\left(-\infty, V_{F}\right]\right)$ such that $v \frac{\partial \phi}{\partial v} \in L^{\infty}\left(\left(-\infty, V_{F}\right)\right)$, the following expression holds in the distributional sense

$$
\frac{d}{d t} \int_{-\infty}^{V_{F}} \phi(v) p(v, t) d v=\int_{-\infty}^{V_{F}}\left[\frac{\partial \phi}{\partial v} h(v, N)+a(N) \frac{\partial^{2} \phi}{\partial v^{2}}\right] p(v, t) d v-N(t) \phi\left(V_{F}\right)+\frac{R(t)}{\tau} \phi\left(V_{R}\right) .
$$

We observe that considering the choice $\phi \equiv 1$ in formulation (66), we obtain the conservation law (3)

$$
R(t)+\int_{-\infty}^{V_{F}} p(v, t) d v=R^{0}+\int_{-\infty}^{V_{F}} p^{0}(v) d v=1
$$

and thus the interpretation as a probability distribution of neurons holds true.

\section{The blow-up result}

In [5] (Theorem 2.2), where refractory states were not taken into account, conditions for finite time blow-up were given. These conditions on the initial data impose that, starting with them, the solutions blow-up in finite time whatever is $b>0$. Here, we present an extension of this result when refractory states are included.

Theorem 3.1 (Finite time blow-up in excitatory case) With the assumptions

$$
h(v, N) \geq b N-v \quad \text { and } \quad a(N) \geq a_{m}>0, \quad-\infty<v \leq V_{F}, \quad 0 \leq N,
$$

a weak solution to the system (III) cannot be global in time (that is, $p(t, v)$ blows-up in finite time) in the two following cases :

1. Fixed initial data. For $p^{0} \neq 0$ fixed, when $b>0$ is large enough.

2. Fixed $b>0$. For $b>0$ fixed, when $p^{0}$ is concentrated enough near $V_{F}$. More precisely, when

$$
\int_{-\infty}^{V_{F}} e^{\mu v} p^{0}(v) d v \geq \frac{e^{\mu v_{F}}}{b \mu}, \quad \text { with } \mu=2 \max \left(\frac{V_{F}}{a_{m}}, \frac{1}{b}\right) .
$$


Proof. With $\mu=2 \max \left(\frac{V_{F}}{a_{m}}, \frac{1}{b}\right)$, we choose the multiplier $\phi(v)=e^{\mu v}$ and define the following exponential moment

$$
M_{\mu}(t)=\int_{-\infty}^{V_{F}} \phi(v) p(v, t) d v
$$

For a weak solution according to (6), we find that

$$
\frac{d}{d t} M_{\mu}(t)=\mu \int_{-\infty}^{V_{F}} h(v, N) \phi(v) p(v, t) d v+\mu^{2} a(N(t)) \int_{-\infty}^{V_{F}} \phi(v) p(v, t) d v-N(t) \phi\left(V_{F}\right)+\frac{R(t)}{\tau} \phi\left(V_{R}\right) .
$$

Therefore, using (17) and the fact that $v \in\left(-\infty, V_{F}\right)$ and $\frac{R(t)}{\tau} \phi\left(V_{R}\right)>0$,

$$
\frac{d}{d t} M_{\mu}(t) \geq N(t)\left[b \mu M_{\mu}(t)-\phi\left(V_{F}\right)\right]+\mu\left(\mu a_{m}-V_{F}\right) M_{\mu}(t)
$$

and finally with the assumption for $\mu$

$$
\frac{d}{d t} M_{\mu}(t) \geq N(t)\left[b \mu M_{\mu}(t)-\phi\left(V_{F}\right)\right]+\mu V_{F} M_{\mu}(t) .
$$

We observe that if the initial state satisfies

$$
b \mu M_{\mu}(t=0) \geq \phi\left(V_{F}\right)
$$

then, using Gronwall's Lemma and the fact that $N(t) \geq 0, M_{\mu}(t)$ increases and stays larger than $\frac{\phi\left(V_{F}\right)}{b \mu}$ for all the time. Therefore (9) can be reduced to

$$
\frac{d}{d t} M_{\mu}(t) \geq \mu V_{F} M_{\mu}(t)
$$

and $M_{\mu}(t) \geq M_{\mu}(t=0) e^{\mu V_{F} t}$ which is a contradiction with the conservation law (3) since

$$
\int_{-\infty}^{V_{F}} p(v, t) d v \leq 1
$$

Consequently to conclude the proof we should show inequality (10).

1. Fixed initial data: Since $M_{\mu}(t=0)$ is fixed, we can choose $b$ large enough such that (10) holds.

2. Fixed $b>0$ : For $b>0$ fixed, if the initial data satisfies (8), then one readily checks that condition (10) holds. There are such initial data, since with our choice of $\mu, b \mu M_{\mu}(t=0) \geq 2 M_{\mu}(t=0)$ and we can also approximate as much as we want by smooth initial probability densities an initial Dirac mass at $V_{F}$, which gives $M_{\mu}(t=0)=\phi\left(V_{F}\right)$.

Remark 3.2 The blow-up cannot be instantaneous for smooth enough initial data because a recent result shows existence of classical solutions for short times, [8]. 


\section{Steady states}

We devote this section to analyze the stationary solutions of the system (11), which are examples of solutions that do not blow-up. To describe the family of steady states, we proceed in analogous way as in [5]. Furthermore, we show that the number of steady states, in terms of the parameters of the system (11), changes with the presence of refractory state. In order to illustrate this situation we also present some numerical results in Section 7.

We write $h(v, N)=v-V_{0}(N)$, then the steady state $(p, N, R)$ of the system (10) satisfies

$$
\frac{\partial}{\partial v}\left[\left(v-V_{0}(N)\right) p+a(N) \frac{\partial}{\partial v} p(v)+\frac{R}{\tau} H\left(v-V_{R}\right)\right]=0, \quad R=\tau N .
$$

The equation on $p$ is understood in the sense of distributions, with $H$ the Heaviside function, i.e., $H(u)=1$ for $u \geq 0$ and $H(u)=0$ for $u<0$. Using the definition of $N$ and the Dirichlet boundary condition in (11), we conclude that

$$
\left(v-V_{0}(N)\right) p+a(N) \frac{\partial p}{\partial v}+\frac{R}{\tau} H\left(v-V_{R}\right)=0, \quad R=\tau N
$$

and therefore,

$$
p(v)=\frac{N}{a(N)} e^{-\frac{\left(v-V_{0}(N)\right)^{2}}{2 a(N)}} \int_{v}^{V_{F}} e^{\frac{\left(w-V_{0}(N)\right)^{2}}{2 a(N)}} H\left[w-V_{R}\right] d w, \quad R=\tau N,
$$

which can be rewritten, using the expression of the Heaviside function as

$$
p(v)=\frac{N}{a(N)} e^{-\frac{\left(v-V_{0}(N)\right)^{2}}{2 a(N)}} \int_{\max \left(v, V_{R}\right)}^{V_{F}} e^{\frac{\left(w-V_{0}(N)\right)^{2}}{2 a(N)}} d w, \quad R=\tau N .
$$

This formula for $p$ is exactly the same as the equation without refractory state. However, to find the stationary value $N$ we need to impose the conservation law (3), which has into account the refractory state,

$$
1-R=\int_{-\infty}^{V_{F}} p(v) d v=\frac{N}{a(N)} \int_{-\infty}^{V_{F}} e^{-\frac{\left(v-V_{0}(N)\right)^{2}}{2 a(N)}} \int_{\max \left(v, V_{R}\right)}^{V_{F}} e^{\frac{\left(w-V_{0}(N)\right)^{2}}{2 a(N)}} d w d v
$$

In this way, we obtain a nonlinear equation for $N$

$$
1-\tau N=\frac{N}{a(N)} \int_{-\infty}^{V_{F}} e^{-\frac{\left(v-V_{0}(N)\right)^{2}}{2 a(N)}} \int_{\max \left(v, V_{R}\right)}^{V_{F}} e^{\frac{\left(w-V_{0}(N)\right)^{2}}{2 a(N)}} d w d v
$$

This equation for $N$ can be rewritten considering the change of variables in []ㅡ:

$$
z=\frac{v-V_{0}}{\sqrt{a}}, \quad u=\frac{w-V_{0}}{\sqrt{a}}, \quad w_{F}=\frac{V_{F}-V_{0}}{\sqrt{a}}, \quad w_{R}=\frac{V_{R}-V_{0}}{\sqrt{a}},
$$

where the dependency upon $N$ has been avoided to simplify notations. In this way the equation (13) is rewritten as

$$
I(N)=\frac{1}{N}-\tau, \quad I(N):=\int_{-\infty}^{w_{F}}\left[e^{-\frac{z^{2}}{2}} \int_{\max \left(z, w_{R}\right)}^{w_{F}} e^{\frac{u^{2}}{2}} d u\right] d z>0,
$$


or, equivalently,

$$
\left\{\begin{array}{l}
N(I(N)+\tau)=1, \\
I(N):=\int_{-\infty}^{w_{F}}\left[e^{-\frac{z^{2}}{2}} \int_{\max \left(z, w_{R}\right)}^{w_{F}} e^{\frac{u^{2}}{2}} d u\right] d z .
\end{array}\right.
$$

Notice that, from (14), the solution obviously satisfies $N<1 / \tau$.

Theorem 4.1 Assume $a(N)=a_{0}$ is constant and $V_{0}(N)=b N$. Then:

- Inhibitory case $(b<0)$ : there is a unique steady state to (10).

- Excitatory case $(b>0)$ : there is a odd number of steady states to (10) (considering multiplicity, see bottom left Figure 11).

This result is different from the case without refractory state where steady states do not always exist and the number can change from odd to even.

Proof. We first recall the following properties for the function $I(N)$, which were proven in [5]:

1. Inhibitory case $(b<0): I(N)$ is an increasing strictly convex function and $\lim _{N \rightarrow \infty} I(N)=\infty$.

2. Excitatory case $(b>0): I(N)$ is a decreasing convex function and $\lim _{N \rightarrow \infty} I(N)=0$.

3. In both cases $(b<0$ and $b>0): I(0)<\infty$.

The result for the inhibitory case $b<0$ is clear. Indeed, checking the relation (14), we notice that $I(0)<\infty, I(N)$ is an increasing strictly convex function and $I(1 / \tau)>0$. Therefore, it crosses to the function $g(N)=\frac{1}{N}-\tau$ at a single point for $N \in[0,1 / \tau]$.

The excitatory case $b>0$ is also clear, since $I(0)<\infty$ and $I(1 / \tau)>0$, while for the function $g(N)=\frac{1}{N}-\tau$ we have $\lim _{N \rightarrow 0} g(N)=\infty$ and $g(1 / \tau)=0$.

Proposition 4.2 For $a_{0}, b>0, V_{R}, V_{F}$ and $\tau$ such that

$$
-I^{\prime}(0)<\tau[I(1 / \tau)+\tau]
$$

there exists a unique steady state to (10).

Condition (16) means that if the refractory period is large enough, there is a unique steady state even in the excitatory case.

Proof. We show that, under the assumption (16), the function $J(N)=N[I(N)+\tau]$ is increasing, that is $J^{\prime}(N)>0$. This is because:

- $J^{\prime}(N)=I(N)+\tau+N I^{\prime}(N)$.

- $I(N)$ is a decreasing function and $I^{\prime}(N)$ is an increasing function, thus, for $0<N<\frac{1}{\tau}$ :

$$
I(1 / \tau)+\tau<I(N)+\tau<I(0)+\tau
$$

and

$$
N I^{\prime}(0)<N I^{\prime}(N)<N I^{\prime}(1 / \tau)
$$


Therefore, $I(1 / \tau)+\tau+N I^{\prime}(0)<J^{\prime}(N)$ and the left hand side in this inequality is positive, if $N<$ $\frac{I(1 / \tau)+\tau}{-I^{\prime}(0)}$. In this way, we conclude the proof, since, if $\frac{1}{\tau}<\frac{I(1 / \tau)+\tau}{-I^{\prime}(0)}$ (condition (16)), this range of values of $N$ is all the range $\left(0, \frac{1}{\tau}\right)$.

Remark 4.3 1. On the other hand, we have just proved that $I(1 / \tau)+\tau+N I^{\prime}(0)<J^{\prime}(N)<I(0)+$ $\tau+N I^{\prime}(1 / \tau)$. Therefore, if $I(0)+\tau+N I^{\prime}(1 / \tau)<0$, we obtain that the function $J$ has at least a maximum (and consequently a minimum), given that $J^{\prime}(0)=I(0)+\tau>0$. The condition

$$
I(0)+\tau+N I^{\prime}(1 / \tau)<0
$$

implies $\frac{I(0)+\tau}{-I^{\prime}(1 / \tau)}<N$. Then, if $\frac{I(0)+\tau}{-I^{\prime}(1 / \tau)}<\frac{1}{\tau}$ there are values of $N$ in the interval $\left[0, \frac{1}{\tau}\right]$ satisfying condition (17) and it holds if

$$
\tau<\tilde{\tau} \quad \text { where } \quad \tilde{\tau}=\frac{-I(0)+\sqrt{I(0)^{2}-4 I^{\prime}(1 / \tau)}}{2} .
$$

In this way, if the value of this maximum is bigger than 1, there are at least three steady states (considering multiplicity). If $J$ has maximum value in $N_{*}$, then

$$
J\left(N_{*}\right)=\frac{\left(I\left(N_{*}\right)+\tau\right)^{2}}{-I^{\prime}\left(N_{*}\right)}=-N_{*}^{2} I^{\prime}\left(N_{*}\right)
$$

which is bigger than 1 if $\frac{1}{\sqrt{-I^{\prime}\left(N_{*}\right)}}<N_{*}$ and there are values of $N \in\left[0, \frac{1}{\tau}\right]$ satisfying it if $\tau<\sqrt{-I^{\prime}(1 / \tau)}$

2. As in [5], analogous results can be stated when $a(N)$ is not constant.

\section{$5 \quad$ Energy for the linear equation and decay rate to the steady state}

For the linear system, which means $b=0$ and $a(N)=a_{0}$, an energy inequality can be written as in $[\underline{5}$. This energy gives the natural space to study the large time relaxation process. To state our result we consider the linear system

$$
\left\{\begin{array}{l}
\frac{\partial p(v, t)}{\partial t}-\frac{\partial}{\partial v}[v p(v, t)]-a_{0} \frac{\partial^{2}}{\partial v^{2}} p(v, t)=\frac{R(t)}{\tau} \delta\left(v-V_{R}\right), \quad v \leq V_{F} \\
\frac{d R(t)}{d t}=N(t)-\frac{R(t)}{\tau} \\
p\left(V_{F}, t\right)=0, \quad N(t):=-a_{0} \frac{\partial}{\partial v} p\left(V_{F}, t\right) \geq 0, \quad a_{0}>0
\end{array}\right.
$$

together with an initial data satisfying

$$
p(v, 0)=p^{0}(v) \geq 0, \quad R(0)=R^{0}>0, \quad \int_{-\infty}^{V_{F}} p^{0}(v) d v+R^{0}=1 .
$$


The unique steady state, as shown in Section 4 , is given by

$$
\left\{\begin{array}{l}
-\frac{\partial}{\partial v}\left[v p_{\infty}(v)\right]-a_{0} \frac{\partial^{2}}{\partial v^{2}} p_{\infty}(v)=\frac{R_{\infty}}{\tau} \delta\left(v-V_{R}\right), \quad v \leq V_{F} \\
p_{\infty}\left(V_{F}\right)=0, \quad N_{\infty}:=-a_{0} \frac{\partial}{\partial v} p_{\infty}\left(V_{F}\right) \geq 0, \quad R_{\infty}=\tau N_{\infty} \\
\int_{-\infty}^{V_{F}} p_{\infty}(v) d v+R_{\infty}=1,
\end{array}\right.
$$

which can be rewritten only in terms of $N_{\infty}$ as follows

$$
\left\{\begin{array}{l}
-\frac{\partial}{\partial v}\left[v p_{\infty}(v)\right]-a_{0} \frac{\partial^{2}}{\partial v^{2}} p_{\infty}(v)=N_{\infty} \delta\left(v-V_{R}\right), \quad v \leq V_{F} \\
p_{\infty}\left(V_{F}\right)=0, \quad N_{\infty}:=-a_{0} \frac{\partial}{\partial v} p_{\infty}\left(V_{F}\right) \geq 0 \\
\int_{-\infty}^{V_{F}} p_{\infty}(v) d v+\tau N_{\infty}=1, \quad R_{\infty}=\tau N_{\infty}
\end{array}\right.
$$

As usual, this system is endowed with a natural energy functional

$$
E(t):=\int_{-\infty}^{V_{F}} \frac{\left(p(v, t)-p_{\infty}(v)\right)^{2}}{p_{\infty}(v)} d v+\frac{\left(R(t)-R_{\infty}\right)^{2}}{R_{\infty}} .
$$

The control of energy dissipation by the two mechanisms of noise and firing allows to prove a rate of convergence to the steady state. Namely, we are going to prove the following theorem.

Theorem 5.1 (Long time behaviour) There is a constant $\nu>0$ which only depends on the model parameters, such that for an initial data with finite energy, there is exponential rate of convergence to the steady state

$$
E(t) \leq E(0) e^{-\nu t}
$$

The proof of this theorem combines a computation of energy dissipation and a Poincaré's inequality. The former is tackled in the following proposition.

Proposition 5.2 (Energy inequality) Solutions to the linear system (18) satisfy

$$
\frac{1}{2} \frac{d}{d t} E(t)=-D_{2}(t) \leq 0
$$

where

$$
D_{2}(t)=a_{0} \int_{-\infty}^{V_{F}} p_{\infty}\left[\frac{\partial}{\partial v}\left(\frac{p}{p_{\infty}}\right)\right]^{2} d v+N_{\infty}\left(\frac{R(t)}{R_{\infty}}-\frac{p\left(V_{R}, t\right)}{p_{\infty}\left(V_{R}\right)}\right)^{2}+N_{\infty}\left(\frac{N(t)}{N_{\infty}}-\frac{R(t)}{R_{\infty}}\right)^{2}
$$

Proof. The energy inequality is a special case of a more general inequality (see [18]) applied to the solution $p(v, t)-p_{\infty}$ of equation (18) and which follows from general relative entropy inequalities [13, 18]. For all convex function $G$ and solution $p(v, t)$ of (18) (whatever is its sign and integral as long as initially the quantities arising in there are well defined) we have

$$
\frac{d}{d t}\left\{\int_{-\infty}^{V_{F}} p_{\infty}(v) G\left(\frac{p(v, t)}{p_{\infty}}\right) d v+R_{\infty} G\left(\frac{R(t)}{R_{\infty}}\right)\right\}=-D_{G}(t) \leq 0,
$$


and $D_{G}(t)$ is given by

$$
\begin{aligned}
D_{G}(t)= & \left.N_{\infty}\left[G\left(\frac{R(t)}{R_{\infty}}\right)-G\left(\frac{p(v, t)}{p_{\infty}(v)}\right)-\left(\frac{R(t)}{R_{\infty}}-\frac{p(v, t)}{p_{\infty}(v)}\right) G^{\prime}\left(\frac{p(v, t)}{p_{\infty}(v)}\right)\right]\right|_{V_{R}} \\
& +a_{0} \int_{-\infty}^{V_{F}} p_{\infty}(v) G^{\prime \prime}\left(\frac{p(v, t)}{p_{\infty}(v)}\right)\left[\frac{\partial}{\partial v}\left(\frac{p(v, t)}{p_{\infty}(v)}\right)\right]^{2} d v \\
& +N_{\infty}\left[G\left(\frac{N(t)}{N_{\infty}}\right)-G\left(\frac{R(t)}{R_{\infty}}\right)-G^{\prime}\left(\frac{R(t)}{R_{\infty}}\right)\left(\frac{N(t)}{N_{\infty}}-\frac{R(t)}{R_{\infty}}\right)\right] \geq 0 .
\end{aligned}
$$

Proposition 5.2 is a consequence of this result with $G(x)=(x-1)^{2}$.

The proof of (23) is a standard computation that we give for the sake of completeness. We compute successively

$$
\begin{aligned}
\frac{\partial}{\partial t} \frac{p}{p_{\infty}}- & \left(v+\frac{2 a_{0}}{p_{\infty}} \frac{\partial}{\partial v} p_{\infty}\right) \frac{\partial}{\partial v} \frac{p}{p_{\infty}}-a_{0} \frac{\partial^{2}}{\partial v^{2}} \frac{p}{p_{\infty}}=\frac{R_{\infty}}{\tau p_{\infty}} \delta\left(v-V_{R}\right)\left(\frac{R}{R_{\infty}}-\frac{p}{p_{\infty}}\right) \\
\frac{\partial}{\partial t} G\left(\frac{p}{p_{\infty}}\right)- & \left(v+\frac{2 a_{0}}{p_{\infty}} \frac{\partial}{\partial v} p_{\infty}\right) \frac{\partial}{\partial v} G\left(\frac{p}{p_{\infty}}\right)-a_{0} \frac{\partial^{2}}{\partial v^{2}} G\left(\frac{p}{p_{\infty}}\right) \\
& =-a_{0} G^{\prime \prime}\left(\frac{p}{p_{\infty}}\right)\left(\frac{\partial}{\partial v} \frac{p}{p_{\infty}}\right)^{2}+\frac{R_{\infty}}{\tau p_{\infty}} \delta\left(v-V_{R}\right)\left(\frac{R}{R_{\infty}}-\frac{p}{p_{\infty}}\right) G^{\prime}\left(\frac{p}{p_{\infty}}\right)
\end{aligned}
$$

and

$$
\begin{aligned}
\frac{\partial}{\partial t} p_{\infty} G & \left(\frac{p}{p_{\infty}}\right)-\frac{\partial}{\partial v}\left[v p_{\infty} G\left(\frac{p}{p_{\infty}}\right)\right]-a_{0} \frac{\partial^{2}}{\partial v^{2}}\left[p_{\infty} G\left(\frac{p}{p_{\infty}}\right)\right] \\
= & -a_{0} p_{\infty} G^{\prime \prime}\left(\frac{p}{p_{\infty}}\right)\left(\frac{\partial}{\partial v} \frac{p}{p_{\infty}}\right)^{2}+\frac{R_{\infty}}{\tau} \delta\left(v-V_{R}\right)\left[\left(\frac{R}{R_{\infty}}-\frac{p}{p_{\infty}}\right) G^{\prime}\left(\frac{p}{p_{\infty}}\right)+G\left(\frac{p}{p_{\infty}}\right)\right] .
\end{aligned}
$$

And we also have

$$
\frac{d}{d t} R_{\infty} G\left(\frac{R}{R_{\infty}}\right)=\frac{R_{\infty}}{\tau} G^{\prime}\left(\frac{R}{R_{\infty}}\right)\left[\frac{N}{N_{\infty}}-\frac{R}{R_{\infty}}\right]
$$

We recall from [5] that, because $p_{\infty}\left(V_{F}\right)=0$ and by l'Hospital rule, we have at $v=V_{F}$,

$$
-a_{0} \frac{\partial}{\partial v}\left[p_{\infty} G\left(\frac{p}{p_{\infty}}\right)\right]=-a_{0} \frac{\partial p_{\infty}}{\partial v} G\left(\frac{p}{p_{\infty}}\right)=N_{\infty} G\left(\frac{N}{N_{\infty}}\right) .
$$

Therefore, integrating the equality (25) and combining it with the latter, we find (23).

To prove exponential rate of convergence to the stationary solution (Theorem [5.1) we need a control of the energy by its energy dissipation. And, as in [5], this is the role of Poincaré's inequality.

Proposition 5.3 (Poincaré's inequality) There is a constant $\nu>0$, which only depends on the model parameters, such that, for

$$
\begin{gathered}
\int_{-\infty}^{V_{F}} p(v) d v+R=1, \\
\nu\left[\int_{-\infty}^{V_{F}} \frac{\left(p-p_{\infty}\right)^{2}}{p_{\infty}} d v+\frac{\left(R-R_{\infty}\right)^{2}}{R_{\infty}}\right] \leq \int_{-\infty}^{V_{F}} p_{\infty}\left[\frac{\partial}{\partial v}\left(\frac{p}{p_{\infty}}\right)\right]^{2} d v+N_{\infty}\left(\frac{R}{R_{\infty}}-\frac{p\left(V_{R}\right)}{p_{\infty}\left(V_{R}\right)}\right)^{2} .
\end{gathered}
$$


The proof of this proposition is given in the Appendix

We now conclude the proof of Theorem [5.1] by an elementary combination of Proposition [5.2 and of Propostion 5.3

\section{Random discharge potential}

It is usual to assume some randomness on the discharge potential. Our next ingredient is to include such an effect, which avoids the blow-up phenomena and generates bounded oscillations representing spontaneous activity of the network. This arises in regimes where, for a deterministic firing potential $V_{F}$, the solution blows-up according to a possible interpretation of the blow-up as a sign of a spontaneous activity in a model that is too rough, [10]. The model we present is a new scenario for describing neuronal phenomena of synchronization of networks and completes those of [3, 4] for NNLIF. In this case, we prove strong controls on the total activity $N(t)$ which discards the blow-up behaviour.

To present the model, we come back to the simpler case where the refractory state is ignored and use a Noisy Integrate and Fire model with variable discharge potential given by

$$
\left\{\begin{array}{l}
\frac{\partial p}{\partial t}+\frac{\partial}{\partial v}[(-v+b N) p]-a(N) \frac{\partial^{2} p}{\partial v^{2}}+\phi_{\varepsilon}(v) p=N(t) \delta\left(v-V_{R}\right), \quad v \in \mathbb{R} \\
N(t)=\int_{-\infty}^{+\infty} \phi_{\varepsilon}(v) p(v, t) d v .
\end{array}\right.
$$

Here the function $\phi_{\varepsilon}(v)$ reprensents the discharge rate at potential $v$ and several variants are possible. For numerical purposes we choose a lipshitzian rate

$$
\phi_{\varepsilon}(v)=\frac{1}{\varepsilon}\left(v-V_{F}\right)_{+} .
$$

Another possible choice is a uniform rate of discharge as soon as $v$ overpasses $V_{F}$, that is, the discontinous function $\phi_{\varepsilon}(v)=\frac{1}{\varepsilon} \mathbb{I}_{\left\{v>V_{F}\right\}}$.

Taking into account the refractory state, the model becomes

$$
\left\{\begin{array}{l}
\frac{\partial p}{\partial t}+\frac{\partial}{\partial v}[(-v+b N) p]-a(N) \frac{\partial^{2} p}{\partial v^{2}}+\phi_{\varepsilon}(v) p=\frac{R(t)}{\tau} \delta\left(v-V_{R}\right), \quad v \in \mathbb{R}, \\
\frac{d R(t)}{d t}=N(t)-\frac{R(t)}{\tau} \\
N(t)=\int_{-\infty}^{+\infty} \phi_{\varepsilon}(v) p(v, t) d v .
\end{array}\right.
$$

This regularization changes the mathematical structure in a fundamental way, since we are now able to prove a priori controls. A simple control is possible when $\phi_{\varepsilon}(v)=\frac{1}{\varepsilon} \mathbb{I}_{\left\{v>V_{F}\right\}}$, then we simply use that $N(t) \leq \frac{1}{\varepsilon} \int_{-\infty}^{+\infty} p(v, t) d v$ and thus $N(t) \leq \frac{1}{\varepsilon}$. For a rate given by (27) we prove global estimates in the following theorem.

Theorem 6.1 (Global estimates) Assume $a(N) \leq a_{0}+a_{2} N^{2}, \int_{-\infty}^{\infty}\left(1+|v|^{3}\right) p^{0}(v) d v<\infty$ and the discharge rate (27), then the solutions to (26) or (28) satisfy the a priori bounds

$$
N(t) \leq \max \left(C, \int_{V_{F}}^{\infty}\left(v-V_{F}\right)^{3} p^{0}(v) d v\right) e^{c t / \varepsilon^{2}} .
$$


Proof. We use the moments defined by $M_{k}(t)=\int_{V_{F}}^{\infty}\left(v-V_{F}\right)^{k} p(v, t) d v$. And, with this notation, we remark that $M_{1}(t)=\varepsilon N(t)$. Then, we compute

$$
\begin{gathered}
\frac{d}{d t} M_{3}(t)+3 \int_{V_{F}}^{\infty}\left(v-V_{F}\right)^{2}(v-b N) p d v-6 a(N) \int_{V_{F}}^{\infty}\left(v-V_{F}\right) p d v+\frac{M_{4}(t)}{\varepsilon}=0, \\
\frac{d}{d t} M_{3}(t)+3 M_{3}(t)+\frac{M_{4}(t)}{\varepsilon} \leq 3\left(\frac{b}{\varepsilon} M_{1}(t)-V_{F}\right) \int_{V_{F}}^{\infty}\left(v-V_{F}\right)^{2} p d v+6\left[a_{0}+\frac{a_{2}}{\varepsilon^{2}} M_{1}(t)^{2}\right] M_{1}(t) .
\end{gathered}
$$

Since $\int_{V_{F}}^{\infty} p(v, t) d v \leq 1$, the Hölder's inequality gives

$$
\begin{aligned}
& M_{1} \leq\left(\int_{V_{F}}^{\infty}\left(v-V_{F}\right)^{3} p(v, t) d v\right)^{1 / 3} \\
& M_{2} \leq\left(\int_{V_{F}}^{\infty}\left(v-V_{F}\right)^{3} p(v, t) d v\right)^{2 / 3} .
\end{aligned}
$$

Therefore, we conclude that

$$
\frac{d}{d t} M_{3}(t)+3 M_{3}(t)+\frac{M_{4}(t)}{\varepsilon} \leq \frac{3 b}{\varepsilon} M_{3}(t)+6 a_{0} M_{3}(t)^{1 / 3}+6 \frac{a_{2}}{\varepsilon^{2}} M_{3}(t) .
$$

The term $M_{3}(t)^{1 / 3}$ makes that the solution may become positive from $t=0$ even for a vanishing initial data $M_{3}(0)$. Then, applying the Gronwall's lemma, we find

$$
M_{3}(t) \leq \max \left(C, M_{3}(0)\right) e^{C t / \varepsilon^{2}}
$$

The result of Theorem 6.1 follows immediately.

Remark 6.2 For the NNLIF model without refractory state and with a deterministic threshold potential, in [7] was given a criterion to determine, in terms of the firing rate $N$, when the weak solutions blow-up in finite time; the a priori estimate $N(t)<\infty$ for all time $t$ is enough for the solution to exist globally. Also [9] give a proof of global existence for a model related to ours with excitatory neurons and jumps rather than a continous drift.

\section{Numerical illustrations and spontaneaous activity}

In order to illustrate our theoretical results and show the behaviour of solutions of NNLIF model, in particular emergence of spontaneous oscillations, we present some numerical illustrations. We consider only the model with refractory state in two cases: with a deterministic firing potential as in (1D) and with randomness on the discharge threshold according to (28). Several times, we have used the same parameter values so that the reader can compare with the numerical results without refractory state in [5].

Our numerical scheme is a simple extension of the method developed in 5. We just take into account the additional ODE for $R(t)$ which does not change the method. For a random discharge threshold the 
computation is run on a large enough interval so as to ensure that the cut-off is negligible due to the exponential absorption for $v>V_{F}$.

\section{Deterministic firing potential}

For a deterministic value of $V_{F}$, we simulate the evolution of solutions for system (11). With these numerical results we can observe the behaviour of this model and the differences with respect to simulations of the NNLIF model without refractory state presented in [5].

In Figure 1 we illustrate the possible multiplicity of steady states. We plot the function $N[I(N)+\tau]$ in (15) and the intersections with the constant 1 (for $N \in[0,1 / \tau)$ ) give the steady states. We plot the curves for different values of the refractory period $\tau$ and the connectivity parameter $b$. Cases with a single or 3 steady states are observed. In particular, for $\tau$ small, the largest root increases which is compatible with the singular limit $\tau=0$ (no refractory state) where there can be an even number of steady states.

Figure 2 shows the distributions $p(v)$ for the three steady states obtained in the top right plot in Figure 1, computed with the analytical formula (12), where $V_{0}(N)=b N$ :

$$
p(v)=\frac{N}{a(N)} e^{-\frac{(v-b N)^{2}}{2 a(N)}} \int_{\max \left(v, V_{R}\right)}^{V_{F}} e^{\frac{(w-b N)^{2}}{2 a(N)}} d w, \quad R=\tau N .
$$

Figure 3 depicts the evolution of $N(t)$ and $R(t)$ for various initial data in the case when there are three steady states, as it was illustrated in Figure 2. In the light of these simulations we can think that the steady states with the least and highest firing rates are stable, while the stationary solution with the medium firing rate is unstable. For this regime of parameters, where three steady states exist, Theorem 3.1 shows that for suitable initial data the solutions blow-up in finite time. Figure 4 describes this situation.

\section{Random distribution of firing potential. Spontaneaous activity}

In our simulations for the system (11) with a deterministic firing potential, we do not find spontaneaous activity, and our numerical results describe evolution to stationary states or blow-up in finite time. A very different situation can happen if a random distribution of firing potential is considered. We have modelled the random distribution of threshold potential by means of the system (28).

In Figure 5 we show the firing rate for the solution to (28), considering distinct values of $V_{R}$ and values of $\epsilon$. In these simulations we observe that oscillations depends on the difference $V_{F}-V_{R}$ and on values of $\epsilon$. More precisely, the results show that for $\epsilon$ fixed, the oscillations increase when $V_{F}-V_{R}$ becomes small and the oscillations disappear if $V_{F}-V_{R}$ is large enough. We also point out the influence of the value of $\epsilon$ in the case where there is no spontaneaous activity. We observe that the maximum value of the firing rate $N$ gets bigger when $\epsilon$ gets smaller.

In these simulations we have used $\phi_{\varepsilon}(v)=\frac{1}{\varepsilon}\left(v-V_{F}\right)_{+}$, but we have checked that similar conclusions hold for other choices of $\phi_{\varepsilon}$.

\section{Conclusion}

One of the simplest models allowing for a self-consistent description of neuronal network is the Network Noisy Leaky Integrate and Fire model. The NNLIF has been widely used in the neuroscience community. 
However, the mathematics behind the model are still poorly studied in particular for the excitatory case. In [5] we started to analyze the mathematical structure of the equation and showed that solutions can blow-up in finite time and described the stationary states of the model. In this paper we have increased the knowledge about NNLIF, since we have included the influence of the refractory state. In this direction we have extended the blow-up results in finite time for a deterministic value $V_{F}$ and we have also shown numerically that spontaneous activity arises in cases where the deterministic value $V_{F}$ is changed to a random discharge potential.

For the excitatory networks with a deterministic value $V_{F}$, the NNLIF with refractory state shares with the model without refractory state the possibility of blow up in finite time, in two ways: for every fixed initial data if the connectivity parameter $b$ is large enough and for any fixed $b$, if the initial data is concentrated enough around the threshold potential $V_{F}$. However, in terms of the set of steady states, the presence of neurons in refractory state can produce more stationary solutions than without refractory state. For instance, for $b=1.5$ in the case without refractory state there are only two steady states, while considering refractory state, with refractory period $\tau=0.025$, there are three.

When the deterministic value $V_{F}$ is changed to a random discharge potential, according to the system (28), spontaneous activity can appear. This phenomenon is not observed on the NNLIF system with a deterministic value $V_{F}$. Our numerical results show the influence of the distance between $V_{R}$ and $V_{F}$ and the value of $\epsilon$ in the system (28) to produce the spontaneous activity.

Therefore, in this paper, we have extended the analytical and numerical knowledge about the NNLIF including the refractory state and describing the circumstances in which spontaneous activity may occur.

To go further in understanding the NNLIF model, several questions are left open: proofs of the stability or unstability of the stationary solutions beyond the linear case related here, analytical study of the system (28) and proof of existence of synchronous states. Another direction is to include the coupling with inhibitory neurons.

Acknowledgements The first author acknowledge support from the project MTM2011-27739-C04-02 (Spain). The authors would like to thank N. Brunel for helpful discussions and references on several aspects of models in neurosciences.

\section{A The poincaré inequality}

This appendix is devoted to the proof of Proposition [5.3. We show that it can be reduced to another inequality derived with recent methods in functional analysis [?].

Before we start with the proof, we warn that, in order to make easier the notation, we denote all the constants by $\mathrm{C}$, which could be different.

We depart from Proposition 4.3 in [5] that we apply to $\tilde{p}_{\infty}=\frac{p_{\infty}}{1-R_{\infty}}$ and $q=p+\tilde{p}_{\infty} R$, which satisfies

$\int_{-\infty}^{V_{F}} q d v=1$. It gives for some constant $C$

$$
\int_{-\infty}^{V_{F}} \tilde{p}_{\infty}(v)\left(\frac{q-\tilde{p}_{\infty}}{\tilde{p}_{\infty}}\right)^{2} d v \leq C \int_{-\infty}^{V_{F}} \tilde{p}_{\infty}\left[\frac{\partial}{\partial v}\left(\frac{q}{\tilde{p}_{\infty}}\right)\right]^{2} d v
$$


and Sobolev imbedding also gives the control

$$
\left(\frac{q-\tilde{p}_{\infty}}{\tilde{p}_{\infty}}\left(V_{R}\right)\right)^{2} \leq C \int_{-\infty}^{V_{F}} \tilde{p}_{\infty}\left[\frac{\partial}{\partial v}\left(\frac{q}{\tilde{p}_{\infty}}\right)\right]^{2} d v
$$

Therefore

$$
\int_{-\infty}^{V_{F}} p_{\infty}\left(\frac{p-p_{\infty}}{p_{\infty}}+\frac{R-R_{\infty}}{1-R_{\infty}}\right)^{2} \leq C \int_{-\infty}^{V_{F}} p_{\infty}\left[\frac{\partial}{\partial v}\left(\frac{p}{p_{\infty}}\right)\right]^{2} d v
$$

and

$$
\left(\frac{p-p_{\infty}}{p_{\infty}}\left(V_{R}\right)+\frac{R-R_{\infty}}{1-R_{\infty}}\right)^{2} \leq C \int_{-\infty}^{V_{F}} p_{\infty}\left[\frac{\partial}{\partial v}\left(\frac{p}{p_{\infty}}\right)\right]^{2} d v .
$$

The first inequality can also be written

$$
\int_{-\infty}^{V_{F}} p_{\infty}\left(\frac{p-p_{\infty}}{p_{\infty}}\right)^{2}-\frac{\left(R-R_{\infty}\right)^{2}}{1-R_{\infty}} \leq C \int_{-\infty}^{V_{F}} p_{\infty}\left[\frac{\partial}{\partial v}\left(\frac{p}{p_{\infty}}\right)\right]^{2} d v
$$

To prove Proposition [5.3, we are reduced to proving that

$$
\left(R-R_{\infty}\right)^{2} \leq C \int_{-\infty}^{V_{F}} p_{\infty}\left[\frac{\partial}{\partial v}\left(\frac{p}{p_{\infty}}\right)\right]^{2} d v+C\left(\frac{R}{R_{\infty}}-\frac{p\left(V_{R}\right)}{p_{\infty}\left(V_{R}\right)}\right)^{2}
$$

or in view of (29), it is enough to prove that

$$
\left(R-R_{\infty}\right)^{2} \leq C\left(\frac{p}{p_{\infty}}\left(V_{R}\right)-1+\frac{R-R_{\infty}}{1-R_{\infty}}\right)^{2}+C\left(\frac{p}{p_{\infty}}\left(V_{R}\right)-1-\frac{R-R_{\infty}}{R_{\infty}}\right)^{2} .
$$

This is an inequality between numbers which is readily checked.

\section{References}

[1] L. F. Aвbоtт And C. V. VReeswiJk, Asynchronous states in networks of pulse-coupled oscillators, Phys. Rev. E, 48 (1993), pp. 1483-1490.

[2] R. Brette And W. Gerstner, Adaptive exponential integrate-and-fire model as an effective description of neural activity, Journal of neurophysiology, 94 (2005), pp. 3637-3642.

[3] N. BRunel, Dynamics of sparsely connected networks of excitatory and inhibitory spiking networks, J. Comp. Neurosci., 8 (2000), pp. 183-208.

[4] N. Brunel and V. Hakim, Fast global oscillations in networks of integrate-and-fire neurons with long firing rates, Neural Computation, 11 (1999), pp. 1621-1671.

[5] M. J. Cáceres, J. A. Carrillo, and B. Perthame, Analysis of nonlinear noisy integrate \& fire neuron models: blow-up and steady states, Journal of Mathematical Neuroscience, 1-7 (2011). 

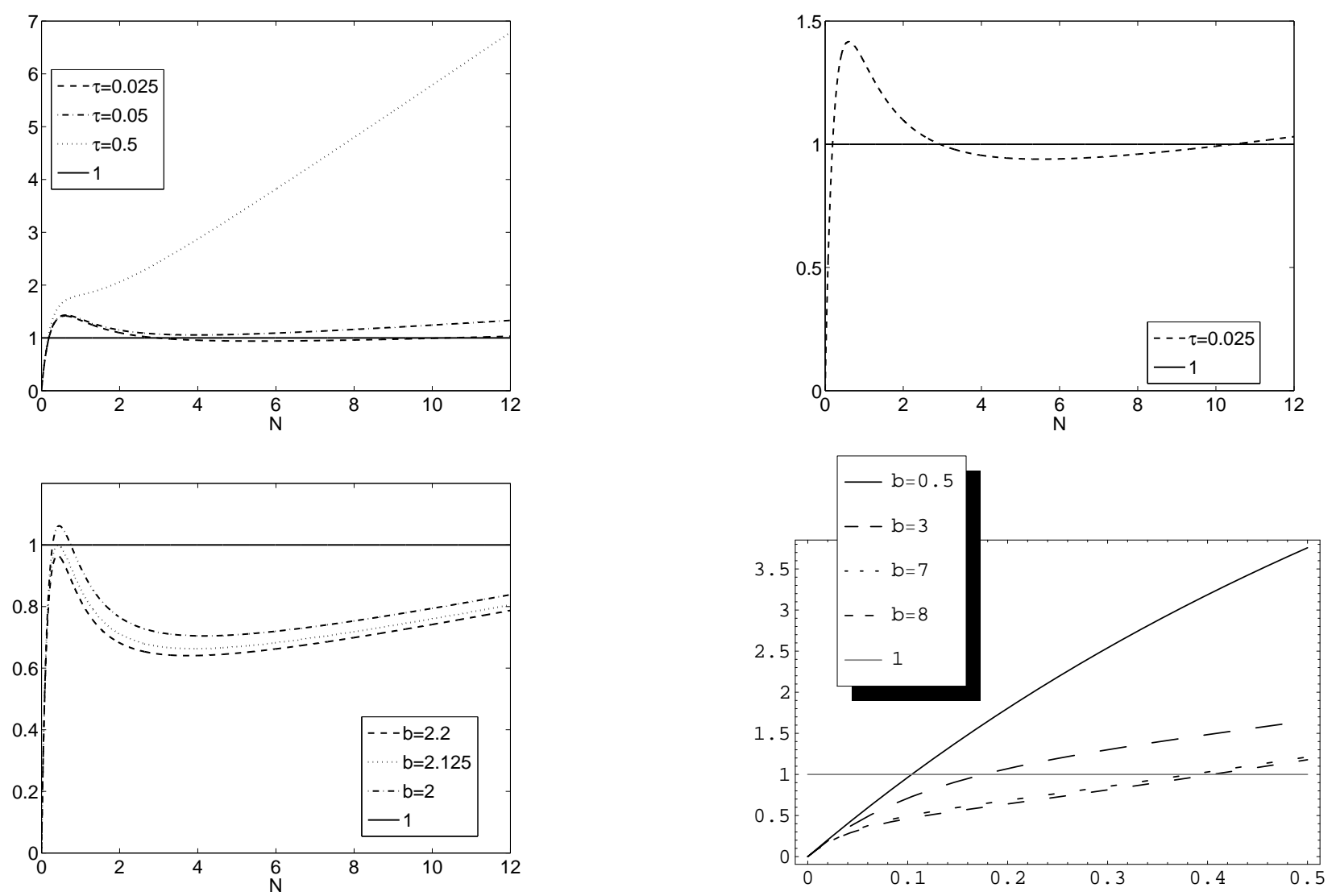

Figure 1: Steady state solution to (11).

Top left figure: the function $N \mapsto N(I(N)+\tau)$ for different values of $\tau$. The solid horizontal line represents the constant 1 . $\left(V_{F}=2, V_{R}=1, a=1\right.$ and $\left.b=1.5\right)$.

Top right figure: Same as left figure with $\tau=0.025$.

Bottom left figure: the function $N \mapsto N(I(N)+\tau)$ for different values of $b$. The solid horizontal line represents the constant 1 . $\left(V_{F}=2, V_{R}=1, a=1\right.$ and $\left.\tau=0.025\right)$.

Bottom right figure: Same as left figure with $V_{F}=2, V_{R}=1, a=1$ and $\tau=2$. 


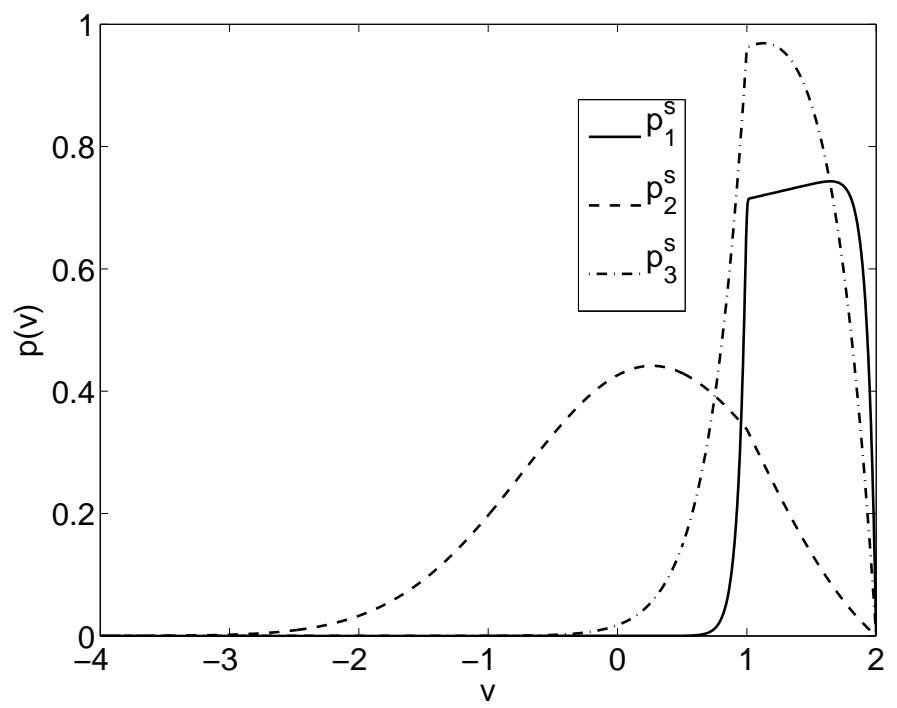

Figure 2: The three stationary states, solutions to (11), when parameters are $V_{F}=2, V_{R}=1, a=1$, $b=1.5$ and $\tau=0.025$.

[6] M. J. Cáceres, J. A. Carrillo, And L. TAO, A numerical solver for a nonlinear fokker-planck equation representation of neuronal network dynamics, J. Comp. Phys., 230 (2011), pp. 1084-1099.

[7] J. A. Carrillo, M. D. M. González, M. P. Gualdani, and M. E. Schonbek, Classical solutions for a nonlinear fokker-planck equation arising in computational neuroscience, Comm. in Partial Differential Equations, 38 (2013), pp. 385-409.

[8] F. Delarue, J. Inglis, S. Rubenthaler, and E. Tanré, Global solvability of a networked integrate-and-fire model of McKean-Vlasov type, 2012. arXiv 1211.0299.

[9] G. Dumont And J. Henry, Population density models of integrate-and-fire neurons with jumps: well-posedness, J. Math. Biol., (2012).

[10] _ Synchronization of an excitatory integrate-and-fire neural network, Bull. Math. Biol., 75 (2013), pp. 629-648.

[11] W. Gerstner and W. Kistler, Spiking neuron models, Cambridge Univ. Press, Cambridge, 2002.

[12] T. Guillamon, An introduction to the mathematics of neural activity, Butl. Soc. Catalana Mat., 19 (2004), pp. 25-45.

[13] P. Michel, S. Mischler, And B. Perthame, General relative entropy inequality: an illustration on growth models, J.Math.Pures Appl., 84 (2005), pp. 1235-1260.

[14] K. Newhall, G. Kovačič, P. Kramer, A. V. Rangan, and D. Cai, Cascade-induced synchrony in stochastically driven neuronal networks, Phys. Rev. E, 82 (2010), p. 041903.

[15] K. Newhall, G. Kovačič, P. Kramer, D. Zhou, A. V. Rangan, and D. Cai, Dynamics of current-based, poisson driven, integrate-and-fire neuronal networks, Comm. in Math. Sci., 8 (2010), pp. 541-600. 

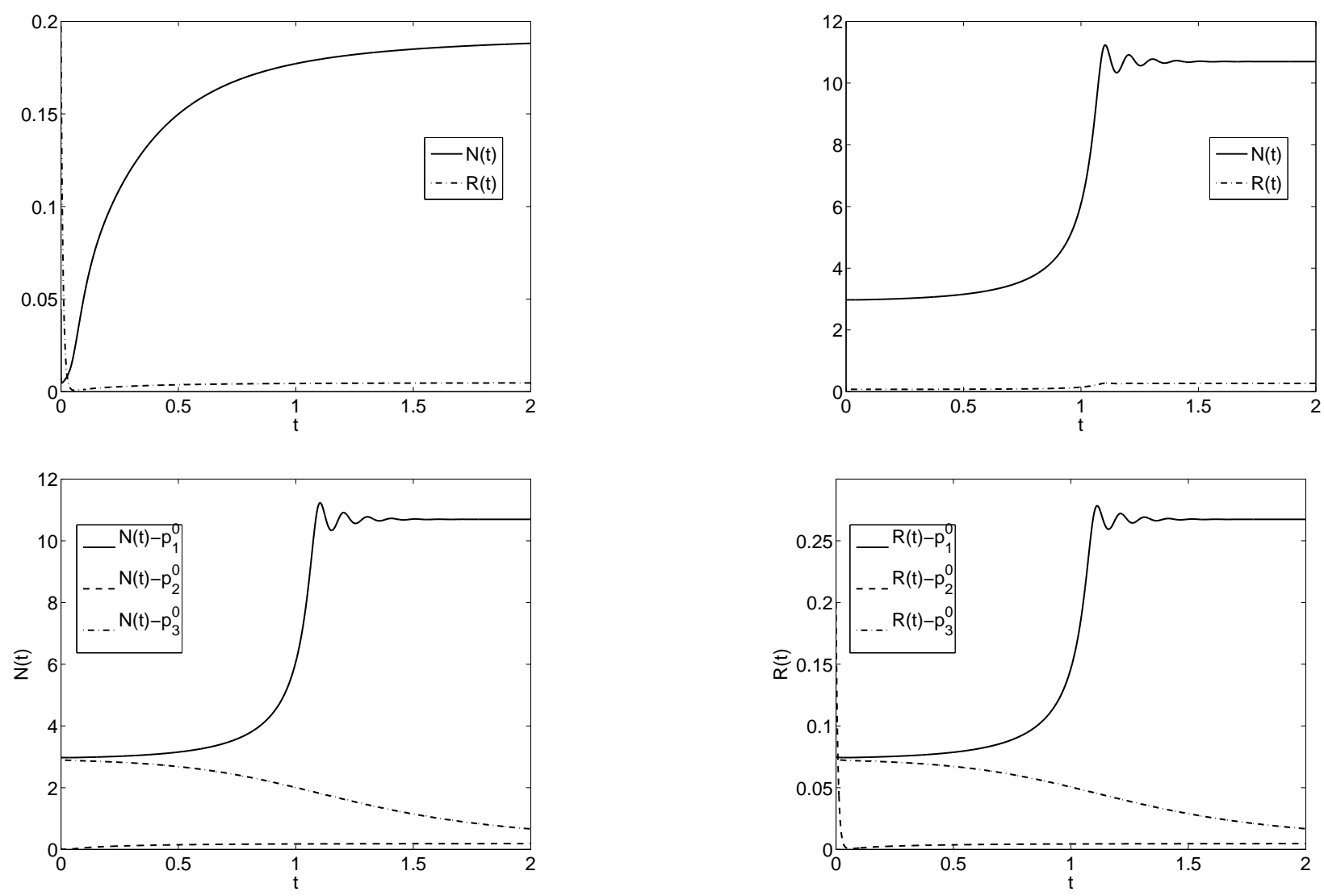

Figure 3: Solution to (1).

Top left figure: Evolution of $R(t)$ and $N(t)$ with the initial data $p^{0}(v)=c e^{-\frac{v^{2}}{0.5}}$ and $R(0)=0.2$, where $c$ is the constant such that $p^{0}$ is a probability distribution, which satisfies (3).

Top right figure: Evolution of $R(t)$ and $N(t)$ where the initial data is the profile (12) with $N=3$ and $R(0)=3 \tau$.

Bottom left figure: Evolution of $N(t)$ for different initial data: $p_{1}^{0}$ is the profile (12) with $N=3$ and $R(0)=N \tau, p_{2}^{0}$ is the profile (12) with $N=2.929$ and $R(0)=N \tau$ and $p_{3}^{0}(v)=c e^{-\frac{v^{2}}{0.5}}$ and $R(0)=0.2$, where $c$ is a constant such that (B) is satisfied.

Bottom right figure: Evolution of $R(t)$ for the three initial data as in the left figure.

In the four plots the other parameters are: $V_{F}=2, V_{R}=1, a=1, b=1.5$ and $\tau=0.025$. 

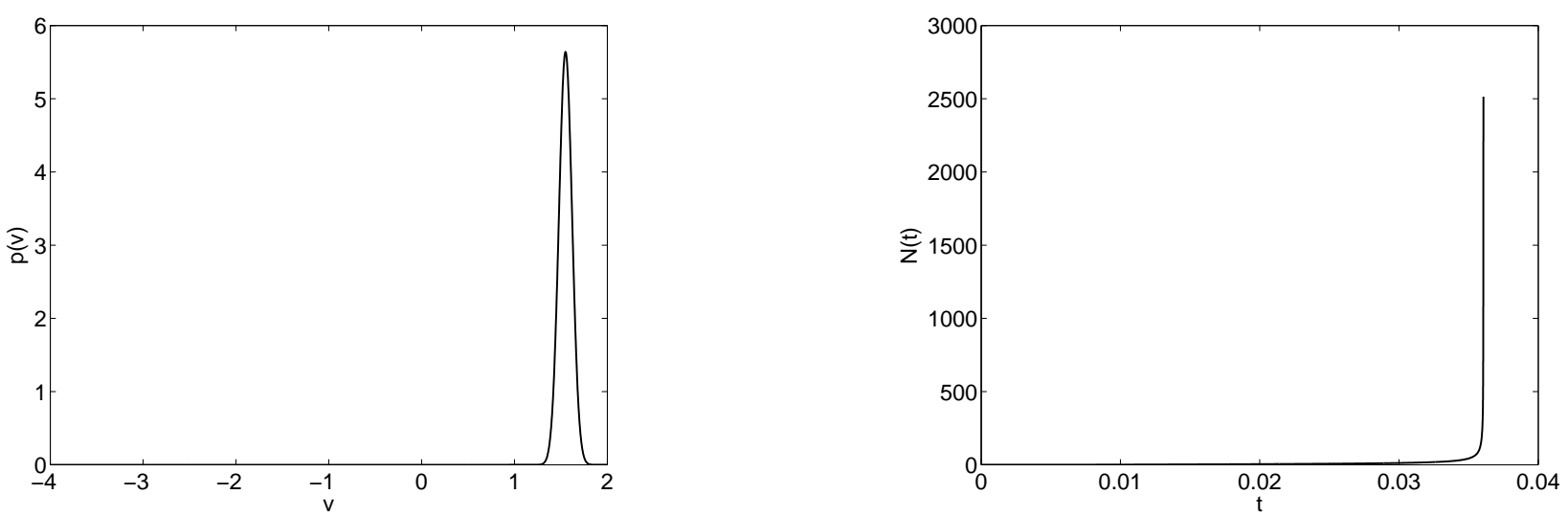

Figure 4: Blowing-up solution to (10). Initial condition (left figure) and evolution of $N(t)$ (right figure) where the solution blows up in finite time. Parameters are $V_{F}=2, V_{R}=1, a=1, b=1.5$ and $\tau=0.025$.
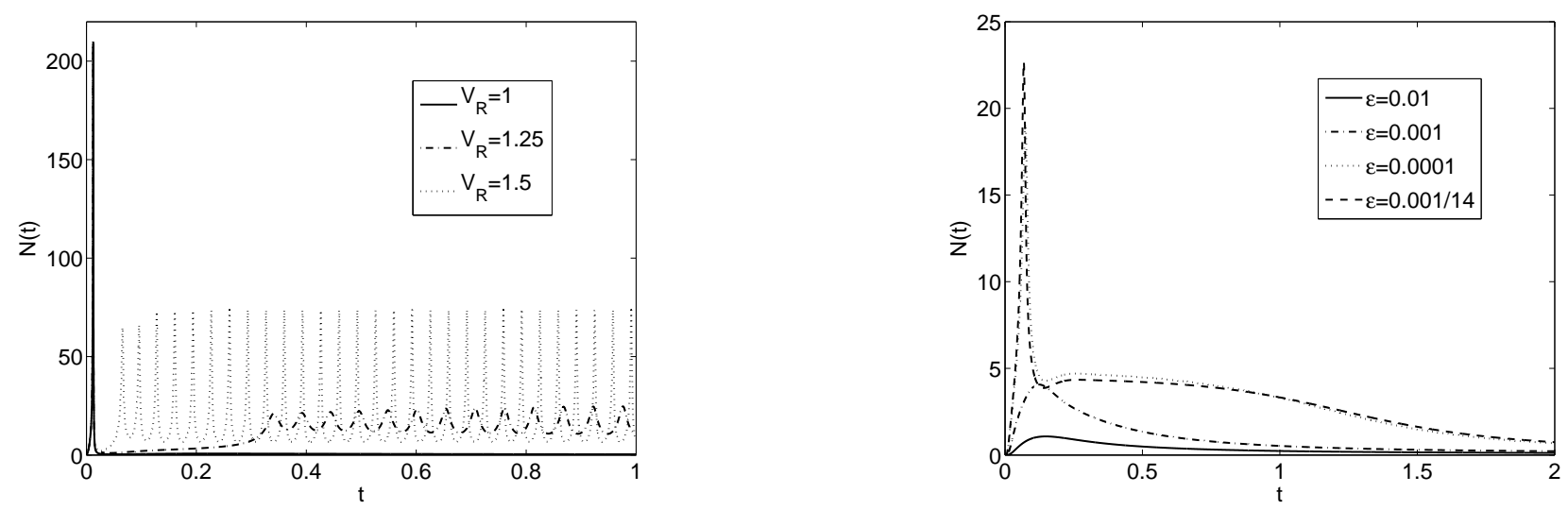

Figure 5: Solution to (28) with $\phi_{\varepsilon}(v)=\frac{1}{\varepsilon}\left(v-V_{F}\right)_{+}$. Left: Evolution of the firing rate $N$ for different values of $V_{R}$. Parameters are $V_{F}=2, a=1, b=1.5, \tau=0.025$ and $\epsilon=1 / 14000$.

Right: Evolution of the firing rate $N$ for different values of $\epsilon$. Parameters are $V_{R}=1, V_{F}=2, a=1$, $b=1.5$ and $\tau=0.025$. 
[16] K. Pakdaman, B. Perthame, and D. Salort, Relaxation and self-sustained oscillations in the time elapsed neuron network model, SIAM J. Appl. MAth., (to appear).

[17] K. Pakdaman, M. Thieullen, and G. Wainrib, Fluid limit theorems for stochastic hybrid systems with application to neuron models, Adv. in Appl. Probab., 42 (2010), pp. 761-794.

[18] B. Perthame, Transport equations in biology, Frontiers in Mathematics, Birkhäuser Verlag, Basel, 2007.

[19] A. V. Rangan, G. KovaC̆IC̆, And D. CAI, Kinetic theory for neuronal networks with fast and slow excitatory conductances driven by the same spike train, Physical Review E, 77 (2008), pp. 1-13.

[20] A. Renart, N. Brunel, And X.-J. Wang, Mean-field theory of irregularly spiking neuronal populations and working memory in recurrent cortical networks, in Computational Neuroscience: A comprehensive approach, J. Feng, ed., Chapman \& Hall/CRC Mathematical Biology and Medicine Series, 2004.

[21] C. Rossant, D. F. M. Goodman, B. Fontaine, J. Platkiewicz, A. K. Magnusson, and R. BRETte, Fitting neuron models to spike trains, Frontiers in Neuroscience, 5 (2011), pp. 1-8.

[22] J. Touboul, Bifurcation analysis of a general class of nonlinear integrate-and-fire neurons, SIAM J. Appl. Math., 68 (2008), pp. 1045-1079.

[23] J. Touboul, Importance of the cutoff value in the quadratic adaptive integrate-and-fire model, Neural Computation, 21 (2009), pp. 2114-2122.

[24] H. Tuckwell, Introduction to Theoretical Neurobiology, Cambridge Univ. Press, Cambridge, 1988. 